\title{
Utility of cytokines to predict neonatal sepsis
}

\author{
Qing Ye', Li-zhong Du' ${ }^{1}$ Wen-Xia Shao² and Shi-qiang Shang'
}

BACKGROUND: Sepsis is an important cause of neonatal morbidity and mortality worldwide. Diagnosis and treatment of neonatal sepsis relies on clinical judgment and interpretation of nonspecific laboratory tests. In a prospective cohort, we measured inflammatory cytokines as a potential biomarker for neonatal sepsis.

METHODS: Serum inflammatory cytokine levels were evaluated in the early stage of neonatal sepsis and after antimicrobial treatment. Receiver operating characteristic curves assessed the diagnostic value of cytokines. We performed multiple logistic regression analysis to characterize the role of each cytokine independently for infants with culture proven sepsis. RESULTS: C-reactive protein, interleukin (IL)-6, IL-10 and IL-6/L-10 levels were significantly elevated in neonatal sepsis when compared with the control group and there were 1.4 (95\% confidence interval (Cl): 1.2-1.5), 4.9 (95\% Cl: 4.6-5.1), 5.1 (95\% Cl: $4.5-5.6)$, and 10.2 (95\% Cl: 9.2-11.1) fold greater odds, respectively, to predict neonatal sepsis when increased. After effective treatment, median IL-6 (pretreatment value: $263.0 \mathrm{pg} /$ $\mathrm{ml}$ and post-treatment value: $7.4 \mathrm{pg} / \mathrm{ml}$ ) and IL-6/L-10 levels (pretreatment value: 16.6 and post-treatment value: 1.4) significantly decreased. The areas under the curve for IL-6, IL-10, IL-6/ IL-10 and C-reactive protein for differential diagnosis were 0.98 , $0.82,0.90$, and 0.88 , respectively.

CONCLUSION: IL-6 and IL-6/IL-10 outperformed C-reactive protein to diagnose neonatal sepsis. Of the cytokines studied, IL-6 was the most sensitive, whereas IL-6/L-10 was the most specific predictor of neonatal sepsis.

D espite recent improvements in maternal and child health outcomes, neonatal sepsis remains an important contributor to infant morbidity and mortality worldwide $(1,2)$. Of the estimated 6.3 million deaths of children $<5$ y of age in 2013, 2.8 million (44\%) occurred during the neonatal period, and 0.42 million (95\% confidence interval $(\mathrm{CI})=0.27-0.69)$ neonatal deaths were due to sepsis (1). Currently, differentiating septic from nonseptic infants is based on nonspecific clinical signs. Prior studies have examined various laboratory markers diagnosis of sepsis. The C-reactive protein (CRP) has been most extensively studied and is often used to distinguish between viral and bacterial infections (3). The CRP is a nonspecific marker for sepsis as it is elevated in certain systemic autoimmune diseases, some oncological diseases, in response to trauma or tissue damage, and after surgery (4). Studies have demonstrated that the specificity and sensitivity of procalcitonin are higher than those of CRP; however, procalcitonin is also not entirely specific to sepsis as its level is elevated in patients who have undergone surgery, in those with florid autoimmune disease and in those who have received an OKT3 monoclonal antibody for immunosuppression after solid organ transplantation (5-7). Routine laboratory tests are ineffective for diagnosing sepsis (8), because they are either nonspecific or have a low sensitivity, often resulting in inappropriate antibiotic use or delayed diagnosis. Sepsis is typically associated with a systemic inflammatory response, with the production and release of a variety of inflammatory mediators, including cytokines $(9,10)$. Therefore, this study aimed to investigate the use of cytokines as diagnostic marker for sepsis.

\section{METHODS}

\section{Participants}

This was a prospective study conducted from January 2011 to December 2015. Ethical approval was obtained from the Institutional Review Board of Zhejiang University (approval number: 2010019). Written informed consent was obtained from all guardians on behalf of the minor/child participants involved in the study. Patients with a positive blood culture and compatible clinical features, including respiratory distress, cyanosis, poor perfusion, lethargy, poor feeding, apnea, and bradycardia were enrolled in the study. At the time of onset of clinical symptoms of neonatal sepsis and before the initiation of any antibiotic therapy, blood samples were collected to measure the serum Th1/Th2 cytokine levels. These indexes were tested again after effective treatment. Effective treatment refers to the disappearance of clinical symptoms and the return of the laboratory indexes to normal levels. Neonatal patients with jaundice $(n=140)$ or enterovirus infection $(n=140)$ and healthy newborns $(n=140)$ were included as controls, and they were matched for age, body weight, and gender. Blood samples were also collected from the neonatal patients with jaundice or enterovirus infection at the time of the onset of clinical symptoms and before the initiation of any antibiotic therapy. Blood samples were collected from the healthy newborns when they came to the hospital for physical examination.

\section{Measurement of Serum Th1/Th2 Cytokine and CRP Levels}

The blood samples were centrifuged at $1,000 \mathrm{~g}$ at $20^{\circ} \mathrm{C}$ for $20 \mathrm{~min}$ after clotting. The serum was carefully harvested, and the Th1/Th2 cytokine levels were measured immediately using 320 flow cytometry. Further, the concentrations of interleukin (IL)-2, IL-4, IL-6, IL-10, tumor necrosis factor (TNF)- $\alpha$, and interferon (IFN)- $\gamma$ were 
determined using a CBA kit (BD CBA Human Th1/Th2 Cytokine Kit II; BD Biosciences, San Jose, CA) (11). The minimum limit of detection for all six cytokines was $1 \mathrm{pg} / \mathrm{ml}$, and the maximum was 5,000 pg/ $\mathrm{ml}$. CRP concentrations were measured using a QuikRead device and QuikRead go CRP kits (Orion Diagnostica, Finland).

\section{Statistical Analysis}

Multiple regression analysis (logistic regression modeling) was performed to examine the independent role of each cytokine, while controlling for all of the others, with culture-proven sepsis used as the outcome variable. The cytokines were used as continuous variables. The odds ratios indicate the time points at which diagnosis of neonatal sepsis was likely vs. the controls for each cytokine evaluated. Comparisons between the study and control groups were performed using the Mann-Whitney $U$-test for continuous variables. All statistical analyses were conducted using SPSS Version 18.0 (PASW Statistics for Windows, Version 18.0. Chicago: SPSS). A $P<0.05$ was considered statistically significant. Further, receiver operating characteristic (ROC) curves were used to assess the diagnostic values of the IL-2, IL-4, IL-6, IL-10, TNF- $\alpha$, IFN- $\gamma$, and CRP levels in discriminating neonatal sepsis. The optimal diagnostic threshold was determined according to Youden's J-statistic, and the relative sensitivity, specificity, positive and negative predictive values were calculated.

\section{RESULTS}

\section{Characteristics of Neonatal Sepsis}

The study group included 420 patients with neonatal sepsis with a male to female ratio of 1.32:1. The risk factors for neonatal sepsis (in order) were as follows: preterm birth, low birth weight, premature rupture of membrane $\geq 18 \mathrm{~h}$, meconiumstained amniotic fluid, neonatal asphyxia and intrauterine fetal distress. Of them, the most important risk factors were preterm delivery and low birth weight. In the study group with neonatal sepsis, $31 \%$ were premature $(n=130)$, and $30.2 \%(n=127)$ had a birth weight of $<2,500 \mathrm{~g}$. Common Clinical manifestations included jaundice, abdominal distension, abnormal temperature, edema, lethargy, apnea, convulsions, and omphalitis. Of these, jaundice (48.1\%) and abdominal distension (39.3\%) were the most common. Gram-positive bacteria accounted for $52.8 \%$ of the pathogenic micro-organisms causing neonatal sepsis, with the main causative organisms being Staphylococcus epidermidis ( $n=87,20.7 \%)$ and S. haemolyticus ( $n=37,8.8 \%)$. In addition, Gram-negative bacteria accounted for $42.2 \%$ of the pathogenic micro-organisms, with the main causative organisms being Escherichia coli $(n=87,20.7 \%)$ and Klebsiella pneumoniae ( $n=64,15.2 \%$ ). Further, fungi accounted for $5 \%$ of the pathogenic micro-organisms causing neonatal sepsis (Table 1).

\section{Th1/Th2 Cytokine and CRP Levels in Neonatal Sepsis}

There were significant differences in the IL-6, IL-10, IL-6/ IL-10, and CRP levels between the study and control groups $(P$ $<0.001)$. However, no difference was found in the IL-2, IL-4, TNF- $\alpha$ or IFN- $\gamma$ level $(P>0.05)$ (Figure 1$)$. The median CRP, IL-6, IL-10, and IL-6/IL-10 levels were significantly elevated in the study group compared with the control group (CRP (mg/ $\mathrm{ml}): 17.0$ vs. $4.0, P<0.001$; IL-6 (pg/ml): 190.7 vs. $3.8, P<$ 0.001 ; IL-10: 10.4 vs. $3.6, P<0.001$; and IL-6/IL-10: 16.6 vs. 1.0 , $P<0.001$ ), with odds ratios of $1.4,4.9,5.1$, and 10.2 , respectively, as determined by multiple regression analysis (logistic regression modeling) performed to examine the independent
Table 1. The features of neonatal sepsis

\begin{tabular}{|c|c|}
\hline Features & Number \\
\hline \multicolumn{2}{|l|}{ Demography } \\
\hline Number & 420 \\
\hline Gender (boys/girls) & $239 / 181$ \\
\hline Gestational age at delivery $<37 \mathrm{wk}$ & $130(31.0 \%)$ \\
\hline Birth weight $<2,500 \mathrm{~g}$ & $127(30.2 \%)$ \\
\hline Asphyxia neonates & $48(11.4 \%)$ \\
\hline Intrauterine fetal distress & $30(7.1 \%)$ \\
\hline Meconium-stained amniotic fluid & $51(12.1 \%)$ \\
\hline Premature rupture of membrane $\geq 18 \mathrm{~h}$ & $60(14.3 \%)$ \\
\hline \multicolumn{2}{|l|}{ Clinic manifestation } \\
\hline Abnormal temperature & $126(30.0 \%)$ \\
\hline Swelling & $43(10.2 \%)$ \\
\hline Lethargy & $142(33.8 \%)$ \\
\hline Omphalitis, pustules or burst of the skin & $52(12.4 \%)$ \\
\hline Vomiting, abdominal distension & $165(39.3 \%)$ \\
\hline Apnea & $60(14.3 \%)$ \\
\hline Jaundice & $202(48.1 \%)$ \\
\hline Seizures & $81(19.3 \%)$ \\
\hline \multicolumn{2}{|l|}{ Complication } \\
\hline Septic stock & $19(4.5 \%)$ \\
\hline Necrotizing enterocolitis & $25(6.0 \%)$ \\
\hline Bacterial meningitis & $61(14.5 \%)$ \\
\hline DIC & $2(0.5 \%)$ \\
\hline \multicolumn{2}{|l|}{ Pathogen } \\
\hline Staphylococcus epidermidis & $87(20.7 \%)$ \\
\hline Staphylococcus haemolyticus & $37(8.8 \%)$ \\
\hline Staphylococcus capitis & $27(6.4 \%)$ \\
\hline Staphylococcus warneri & $18(4.3 \%)$ \\
\hline Staphylococcus aureus & $21(5.0 \%)$ \\
\hline Enterococci & $32(7.6 \%)$ \\
\hline Klebsiella pneumoniae & $64(15.2 \%)$ \\
\hline Escherichia coli & $87(20.7 \%)$ \\
\hline Acinetobacter baumannii & $26(6.2 \%)$ \\
\hline Candida & $21(5.0 \%)$ \\
\hline
\end{tabular}

role of each cytokine, while controlling for all of the others, with culture proven sepsis used as the outcome variable. After effective treatment, the median IL- 6 and IL-6/IL-10 levels were significantly decreased (IL-6 (pg/ml): 263.0 vs. $7.4, P$ $<0.001$; and IL-6/IL-10: 16.6 vs. $1.4, P<0.001$ ) (Figure 2). There were significant differences in the IL-10 and IFN- $\gamma$ levels between early-onset sepsis and late-onset sepsis $(P<0.05)$, but no differences were found in the IL-2, IL-4, IL-6, IL-6/IL-10 or TNF- $\alpha$ level $(P>0.05)$. Further, there were no significant differences in the serum cytokine levels between the neonatal septic patients with Gram-positive bacteria and those with Gram-negative bacteria $(P>0.05)$. Detailed information is provided in Table 2. 


\section{Articles $\mid$ Ye etal.}

a

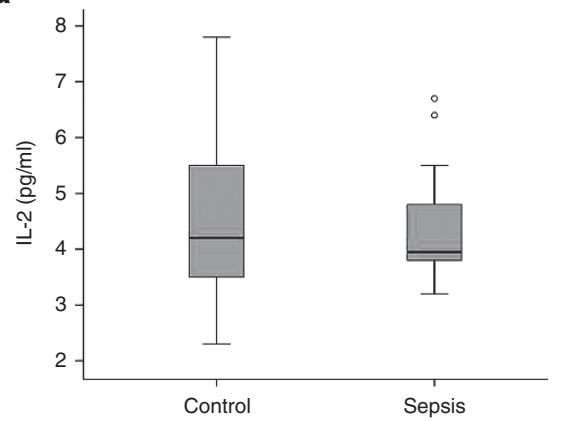

d

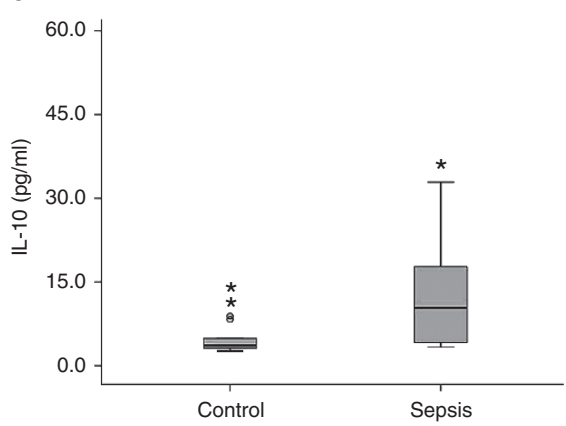

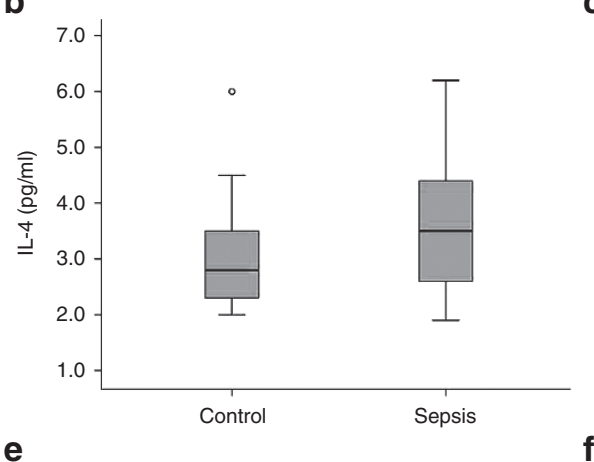

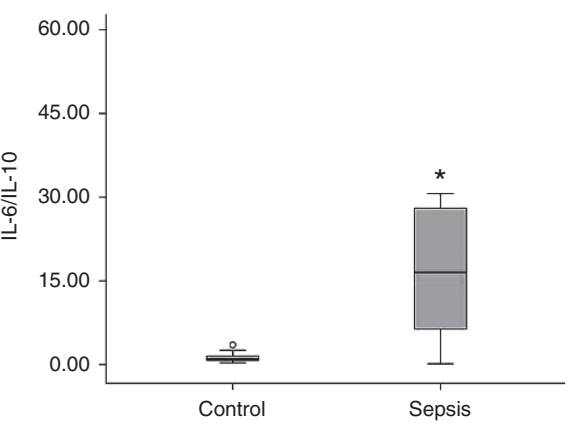

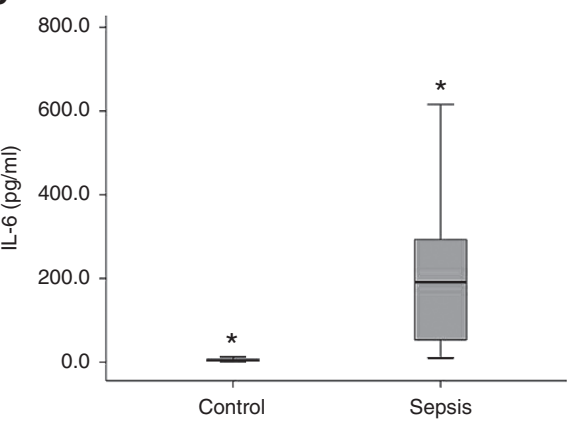

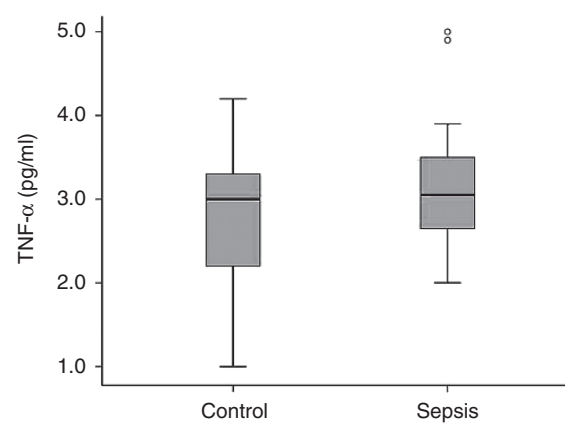

g

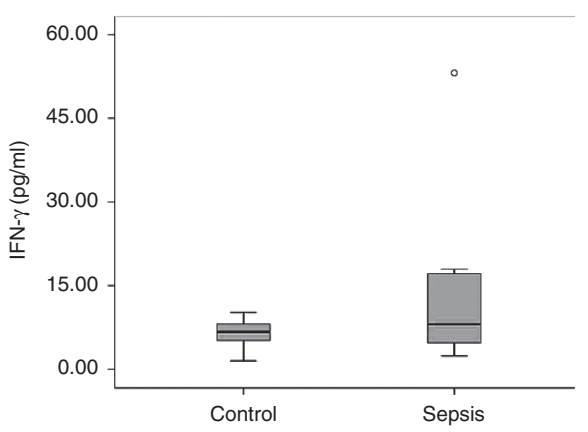

h

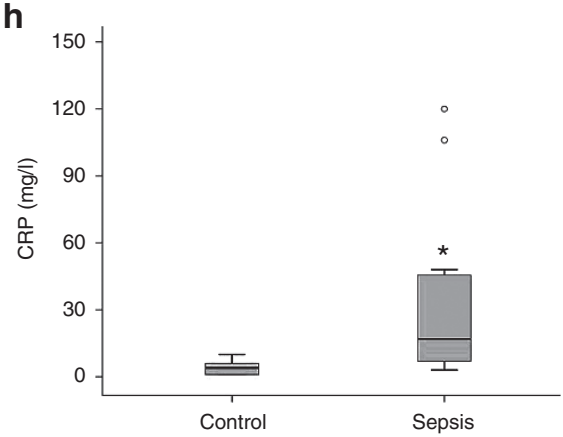

Figure 1. Serum concentrations of inflammatory cytokines and C-reactive protein in neonatal sepsis. (a) IL-2 level. (b) IL-4 level. (c) IL-6 level: ${ }^{*} P<0.001$ vs. control group. (d) IL-10 level: ${ }^{*}<<0.001$ vs. control group. (e) IL-6/L-10 level: ${ }^{*}<0.001$ vs. control group. (f) TNF- $\alpha$ level. (g) IFN- $\gamma$ level. (h) CRP level: ${ }^{*} P<0.001$ vs. control group. CRP, C-reactive protein; IFN- $\gamma$, interferon- $\gamma$; IL-2, interleukin; TNF- $\alpha$, tumor necrosis factor- $\alpha$.

a

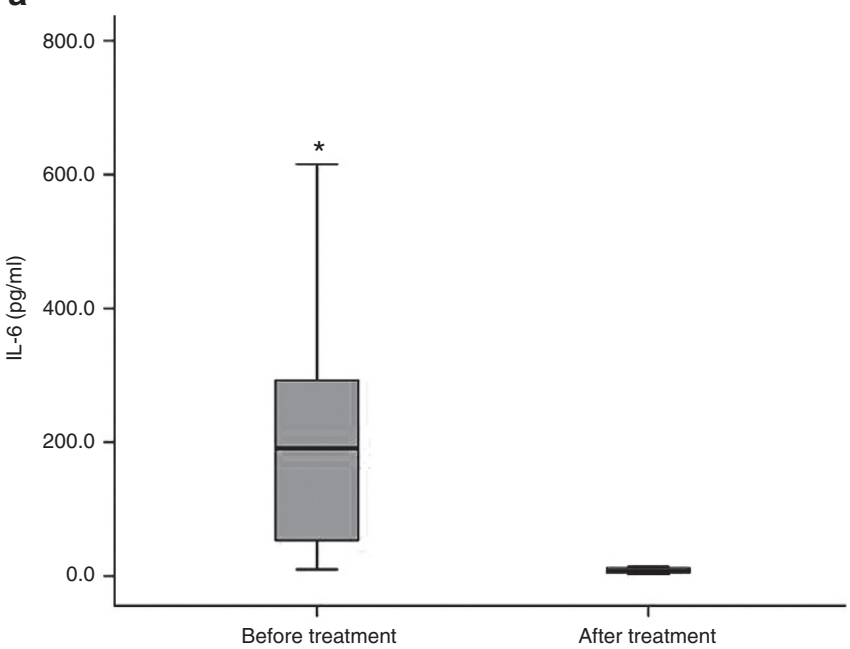

b

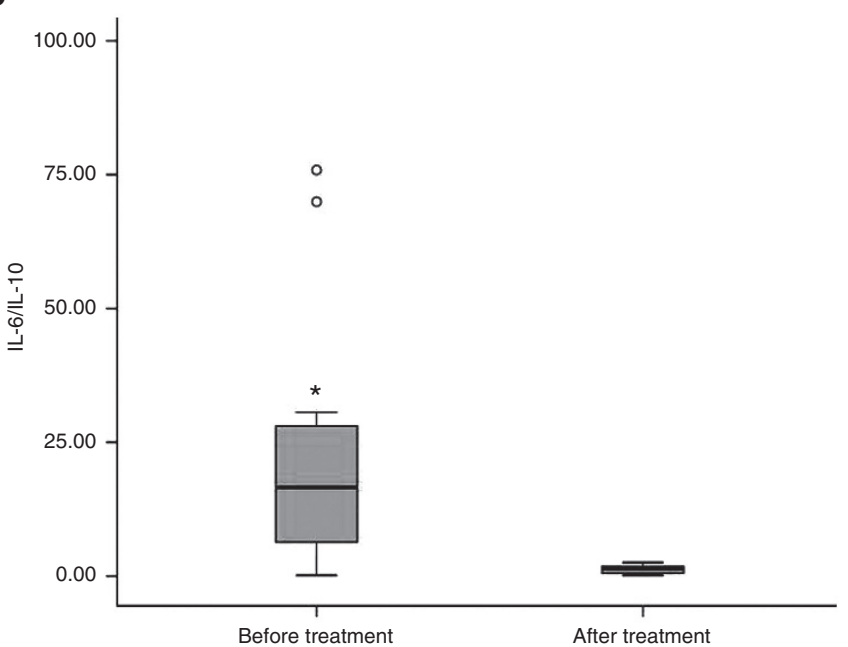

Figure 2. Changes in IL-6 level and IL-6/IL-10 ratio before and after effective treatment for neonatal sepsis. (a) IL-6 level: ${ }^{*} P<0.001$ vs. after effective treatment. (b) IL-6/IL-10 level: ${ }^{*} P<0.001$ vs. after effective treatment. IL, interleukin. 
Using Inflammatory Cytokine Levels to Identify Neonatal Sepsis The results of this study revealed that the development of sepsis resulted in significant alterations in the CRP, IL-6, IL-10, and IL-6/IL-10 levels, with larger odds ratios compared with other diagnostic indicators; therefore, we evaluated the use of these indicators in the differential diagnosis of neonatal sepsis. For the differential diagnosis of this condition, the areas under the ROC curves were 0.98 for IL-6, 0.82 for IL-10, 0.90 for IL-6/IL-10, and 0.88 for CRP. A serum IL-6 level of $>12.5$ $\mathrm{pg} / \mathrm{ml}$ had a sensitivity of $93.75 \%$ and a specificity of $94.12 \%$ in the differential diagnosis of neonatal sepsis; in addition, a serum IL-10 level of $>3.8 \mathrm{pg} / \mathrm{ml}$ had a sensitivity of $87.50 \%$ and a specificity of $61.76 \%$, and a serum IL-6/IL-10 level of $>3.5$ had a sensitivity of $81.25 \%$ and a specificity of $100 \%$. In addition, at a CRP level of $>10.0 \mathrm{mg} / \mathrm{l}$, the diagnostic sensitivity was $62.50 \%$, and the specificity was $100 \%$. Detailed patient information is provided in Table 3 and Figure 3.

\section{DISCUSSION}

The most reliable diagnostic tool for neonatal sepsis, often referred to as the gold standard is a blood culture test for bacteria. While this test is the most reliable diagnostic tool that is currently available, it can take up to $48 \mathrm{~h}$ to obtain results, and treatment must often begin before the results are known. An additional complication is that this test can yield negative results for one out of five patients with sepsis $(12,13)$. Therefore, identifying new biomarkers is critically important.

Diagnostic hematological biomarkers, such as CRP, have been previously studied (14), and while they have been shown to be correlated with sepsis, they have been considered to have limited diagnostic potential $(15,16)$. Infectious and noninfectious diseases, such as malignancies and inflammatory diseases can also cause CRP production (17). Sepsis development can begin following the recognition of one or more components of an invading organism, including structural elements, such as Gram-negative endotoxins and secreted exotoxins, which stimulate the local and systemic release of endogenous inflammatory mediators. These inflammatory mediators include cytokines, such as TNF- $\alpha$, IFN- $\gamma$, and IL-6, which promote the migration and activation of immune cells $(18,19)$. Therefore, this study focused on the efficacy of cytokines for the early diagnosis of sepsis. Although IL-2, IL-4, TNF- $\alpha$, and IFN$\gamma$ have been implicated as major mediators of sepsis, no significant differences in their levels were found compared with the control group in this study. Among these cytokines, IL-4 is considered the most relevant for mycoplasma pneumoniae infection, and the IFN- $\gamma$ level is higher in viral infections $(20,21)$.

Our results showed that IL-6 and IL-6/IL-10 were more effective than CRP in the differential diagnosis of neonatal sepsis.

Table 2. Serum cytokine levels in early-onset sepsis, late-onset sepsis and neonatal sepsis patients with gram-positive bacteria or gram-negative bacteria

\begin{tabular}{lcccccccc}
\hline & & & & & \multicolumn{2}{c}{ Gram-positive } & \multicolumn{2}{c}{ Gram-negative } \\
Diagnostic indicators & Early-onset sepsis & Late-onset sepsis & $Z$ & $P$ & bacteria & bacteria & Z & $P$ \\
\hline IL-2 $(\mathrm{pg} / \mathrm{ml})$ & $3.9(4.0-5.0)$ & $4.0(3.0-7.0)$ & -0.877 & 0.380 & $4.0(3.0-6.0)$ & $5.2(3.0-7.0)$ & 0.463 & 0.521 \\
IL-4 $(\mathrm{pg} / \mathrm{ml})$ & $4.0(3.4-4.4)$ & $3.3(1.9-6.2)$ & -0.721 & 0.471 & $3.7(2.1-4.6)$ & $2.7(1.9-6.2)$ & 0.331 & 0.379 \\
IL-6 $(\mathrm{pg} / \mathrm{ml})$ & $2,007.7(38.0-5,045.0)$ & $180.6(9.7-615.5)$ & -1.277 & 0.201 & $170.4(9.7-5,045.0)$ & $219.6(64.8-615.5)$ & 0.396 & 0.446 \\
IL-10 $(\mathrm{pg} / \mathrm{ml})$ & $4.1(3.3-11.9)$ & $11.9(3.6-213.9)$ & -2.354 & 0.019 & $8.7(3.3-80.5)$ & $20.9(5.9-213.9)$ & 0.182 & 0.212 \\
IL-6/lL-10 & $16.9(11.5-1,230.5)$ & $16.3(0.1-75.9)$ & -0.256 & 0.798 & $16.6(0.1-1,230.4)$ & $16.3(1.2-69.9)$ & 0.808 & 0.862 \\
TNF-a (pg/ml) & $3.5(3.0-4.9)$ & $2.9(2.0-5.0)$ & -1.138 & 0.255 & $3.3(2.0-4.9)$ & $2.7(2.5-5.0)$ & 0.463 & 0.521 \\
IFN- $\gamma(\mathrm{pg} / \mathrm{ml})$ & $9.9(4.0-109.0)$ & $6.6(2.4-305.8)$ & -2.975 & 0.003 & $9.7(2.4-305.8)$ & $6.0(4.1-16.3)$ & 0.467 & 0.521 \\
\hline
\end{tabular}

IL-1, interleukin-1; IFN- $\gamma$, interferon- $\gamma$; TNF- $\alpha$, tumor necrosis factor- $\alpha$.

Table 3. Performances of inflammatory cytokines in discriminating neonatal sepsis

\begin{tabular}{|c|c|c|c|c|c|c|c|c|c|c|c|c|}
\hline Diagnostic indicators & Neonatal sepsis & Control & $P$ & AUC & $\begin{array}{l}\text { Youden } \\
\text { index J }\end{array}$ & Cut off & $\begin{array}{c}\text { Sensitivity } \\
(\%)\end{array}$ & $\begin{array}{c}\text { Specificity } \\
(\%)\end{array}$ & $\begin{array}{l}\text { PPV } \\
(\%)\end{array}$ & $\begin{array}{l}\text { NPV } \\
(\%)\end{array}$ & $\begin{array}{l}\text { Odds } \\
\text { ratios }\end{array}$ & $95 \% \mathrm{Cl}$ \\
\hline IL-2 (pg/ml) & $4.0(3.0-7.0)$ & $4.2(2.0-8.0)$ & 0.925 & 0.50 & 0.081 & $>4.0$ & 37.50 & 70.59 & 37.5 & 70.6 & 0.9 & $0.8-1.0$ \\
\hline IL-6 (pg/ml) & $190.7(9.7-5,045.0)$ & $3.8(1.1-42.4)$ & $<0.001$ & 0.98 & 0.879 & $>12.5$ & 93.75 & 94.12 & 88.2 & 97.0 & 4.9 & $4.6-5.1$ \\
\hline IL-10 (pg/ml) & $10.4(3.3-213.9)$ & $3.6(2.6-12.0)$ & $<0.001$ & 0.82 & 0.493 & $>3.8$ & 87.50 & 61.76 & 51.9 & 91.3 & 5.1 & $4.5-5.6$ \\
\hline $\mathrm{IFN}-\gamma(\mathrm{pg} / \mathrm{ml})$ & $8.1(2.4-305.8)$ & $6.7(1.5-10.2)$ & 0.197 & 0.61 & 0.471 & $>9.3$ & 50.00 & 97.10 & 88.9 & 80.5 & 1.2 & $1.1-1.4$ \\
\hline $\mathrm{CRP}(\mathrm{mg} / \mathrm{l})$ & $17.0(3.0-256.0)$ & $4.0(1.0-10.0)$ & $<0.001$ & 0.88 & 0.625 & $>10.0$ & 62.50 & 100.00 & 100.0 & 85.0 & 1.4 & $1.2-1.5$ \\
\hline
\end{tabular}

AUC, area under the curve; $\mathrm{Cl}$, confidence interval; CRP, C-reactive protein; IL-1, interleukin-1; IFN- $\gamma$, interferon- $\gamma$; NPV, negative predictive value; TNF- $\alpha$, tumor necrosis factor- $\alpha$; PPV, positive predictive value.In this study, multiple regression analysis (logistic regression modeling) was used to explore the independent role of each cytokine controlling for all the others with culture proven sepsis as the outcome variable. Odds ratios show the time points at which diagnosis of neonatal sepsis was likely vs. the controls for each cytokine evaluated. 


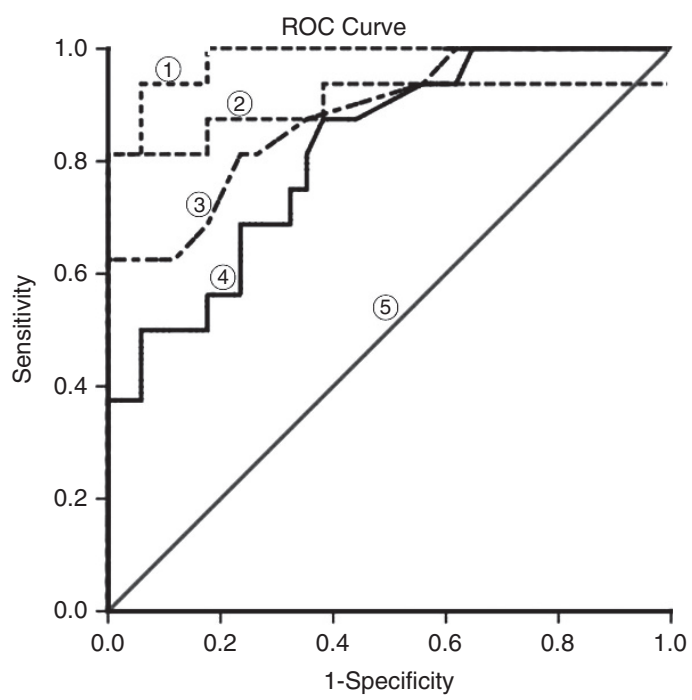

Figure 3. Receiver operating characteristic curve analysis evaluating the use of cytokines in discriminating neonatal sepsis. AUC, area under the curve. (1) ROC of IL- 6 , with an AUC of 0.98 ; (2) ROC of IL6/10, with an AUC of 0.90 ; (3) ROC of CRP, with an AUC of 0.88 ; (4) ROC of IL-10, with an AUC of 0.82 ; and (5) reference line. CRP, C-reactive protein; IL-6, interleukin-6; ROC, receiver operating characteristic curve.

IL-6 had the highest diagnostic sensitivity, whereas IL-6/IL-10 had the best diagnostic specificity. These findings are consistent with those of previous studies $(11,22,23)$. However, to better simulate the actual clinical situation and increase the relevance of the results, our study included a control group consisting of newborns with neonatal jaundice and intestinal viruses, which are common and can be easily confused with neonatal sepsis. The sample size of this study was also larger than those of previous studies; thus, our findings may be of greater reference value.

IL-6 is a cytokine involved in the early host response to infection preceding the increase in CRP. It is synthesized by endothelial cells, mononuclear phagocytes, fibroblasts, amnion, and trophoblastic cells shortly after stimulation by microbial products (24). As the IL-6 level is higher than the CRP level in early disease stages, IL-6 may be useful for early diagnosis (25). In vivo experiments have shown that after lipopolysaccharide injection, the release of IL- 6 occurs at $1-2 \mathrm{~h}$, the peak response occurs at $3 \mathrm{~h}$, and then its level decreases to the baseline level at $8 \mathrm{~h}$ (26). The half-life of IL-6 is $~ 100 \mathrm{~min}$ (27), and its circulating concentration can drop precipitously following antimicrobial treatment (28). The body's immune response pathway is different in the presence of a viral infection compared with that in the presence of a bacterial infection, although elevations in the IL- 6 and CRP levels occur in virally infected children but are generally less pronounced (3). Our findings also showed that after the neonatal sepsis patients received effective treatment, their IL-6 levels dropped significantly, suggesting that IL- 6 can be used as a therapeutic monitoring parameter. However, in some cases, such as chorioamnionitis, the passive transfer of IL- 6 can result in an elevated IL-6 level after birth, which may lead to a false-positive result. Thus, it is necessary to assess maternal risk factors.
IL-10 is an anti-inflammatory cytokine released by monocytes, macrophages, $\mathrm{T}$ and $\mathrm{B}$ lymphocytes, neutrophils, and mesangial cells. Because IL- 6 and IL-10 are two indicators associated with sepsis, the IL-6/IL-10 ratio has higher specificity in the differential diagnosis of neonatal sepsis. In addition, the appropriate response of IL-10 may have a protective effect on systemic inflammatory response syndrome, and a high IL-6/IL-10 ratio has been observed in patients with a worse prognosis (28).

Flow cytometry is an efficient method for detecting cytokines and may be useful in some medical settings; but is not routinely available in most laboratories. Other cytokine detection methods, such as ELISA, are currently available. The reagent cost associated with ELISA is low, and it does not require the use of expensive instruments; therefore, this technique can be used in hospitals at various levels.

In conclusion, the results of this study demonstrated that levels of IL-6 level and IL-6/IL-10 are more sensitive and specific for diagnosing neonatal sepsis than CRP. IL-6 had the highest diagnostic sensitivity, whereas IL-6/IL-10 had the greatest diagnostic specificity. IL-6/IL-10 may be a marker for effective antimicrobial treatment in neonatal sepsis. Biomarkers do not replace clinical judgment in evaluating and empirically treating neonatal sepsis; rather, analysis of cytokines should serve to support clinical decision-making while awaiting definitive test results.

\section{STATEMENT OF FINANCIAL SUPPORT}

This project was supported by grants from the National Natural Science Foundation of China (Grant No. 81501760), Zhejiang Provincial Natural Science Foundation of China (Grant No. LQ16H050002), Zhejiang Provincial Healthy Science Foundation of China (Grant No. 2015KYB191). The funders did not take part in the study.

Disclosure: No authors have conflicts of interest to declare.

\section{REFERENCES}

1. Liu L, Oza S, Hogan D, et al. Global, regional, and national causes of child mortality in 2000-13, with projections to inform post-2015 priorities: an updated systematic analysis. Lancet 2015;385:430-40.

2. Lawn JE, Blencowe H, Oza S, et al.; Lancet Every Newborn Study Group. Every newborn: progress, priorities, and potential beyond survival. Lancet 2014;384:189-205.

3. Gendrel D, Raymond J, Coste J, et al. Comparison of procalcitonin with C-reactive protein, interleukin 6 and interferon-alpha for differentiation of bacterial vs. viral infections. Pediatr Infect Dis J 1999;18:875-81.

4. Ansar W, Ghosh S. C-reactive protein and the biology of disease. Immunol Res 2013;56:131-42.

5. Molter GP, Soltész S, Kottke R, Wilhelm W, Biedler A, Silomon M. [Procalcitonin plasma concentrations and systemic inflammatory response following different types of surgery]. Anaesthesist 2003;52:210-7.

6. Buhaescu I, Yood RA, Izzedine H. Serum procalcitonin in systemic autoimmune diseases-where are we now? Semin Arthritis Rheum 2010;40: 176-83.

7. Zazula R, Prucha M, Tyll T, Kieslichova E. Induction of procalcitonin in liver transplant patients treated with anti-thymocyte globulin. Crit Care 2007;11:R131.

8. Edgar JD, Gabriel V, Gallimore JR, McMillan SA, Grant J. A prospective study of the sensitivity, specificity and diagnostic performance of soluble intercellular adhesion molecule 1, highly sensitive C-reactive protein, soluble E-selectin and serum amyloid $\mathrm{A}$ in the diagnosis of neonatal infection. BMC Pediatr 2010;10:22.

9. Jacobi J. Pathophysiology of sepsis. Am J Health Syst Pharm 2002;59 Suppl $1: S 3-8$. 
10. Kenzel S, Henneke P. The innate immune system and its relevance to neonatal sepsis. Curr Opin Infect Dis 2006;19:264-70.

11. Ye Q, Shao WX, Xu XJ, Yang YZ. The clinical application value of cytokines in treating infectious diseases. PLoS One 2014;9:e98745.

12. Ganatra HA, Stoll BJ, Zaidi AK. International perspective on early-onset neonatal sepsis. Clin Perinatol 2010;37:501-23.

13. Benitz WE. Adjunct laboratory tests in the diagnosis of early-onset neonatal sepsis. Clin Perinatol 2010;37:421-38.

14. Ng PC, Cheng SH, Chui KM, et al. Diagnosis of late onset neonatal sepsis with cytokines, adhesion molecule, and C-reactive protein in preterm very low birthweight infants. Arch Dis Child Fetal Neonatal Ed 1997;77:F221-7.

15. Pierrakos C, Vincent JL. Sepsis biomarkers: a review. Crit Care 2010;14:R15.

16. Malik A, Hui CP, Pennie RA, Kirpalani H. Beyond the complete blood cell count and C-reactive protein: a systematic review of modern diagnostic tests for neonatal sepsis. Arch Pediatr Adolesc Med 2003;157:511-6.

17. Kao PC, Shiesh SC, Wu TJ. Serum C-reactive protein as a marker for wellness assessment. Ann Clin Lab Sci 2006;36:163-9.

18. Bayston KF, Cohen J. Bacterial endotoxin and current concepts in the diagnosis and treatment of endotoxaemia. J Med Microbiol 1990;31:73-83.

19. Bone RC, Grodzin CJ, Balk RA. Sepsis: a new hypothesis for pathogenesis of the disease process. Chest 1997;112:235-43.

20. Ye Q, Xu XJ, Shao WX, Pan YX, Chen XJ. Mycoplasma pneumoniae infection in children is a risk factor for developing allergic diseases. ScientificWorldJournal 2014;2014:986527.
21. Ye Q, Shao WX, Shang SQ, Pan YX, Shen HQ, Chen XJ. Epidemiological characteristics and immune status of children with Respiratory Syncytial Virus. J Med Virol 2015;87:323-9.

22. Sugitharini V, Prema A, Berla Thangam E. Inflammatory mediators of systemic inflammation in neonatal sepsis. Inflamm Res 2013;62: 1025-34.

23. Delanghe JR, Speeckaert MM. Translational research and biomarkers in neonatal sepsis. Clin Chim Acta 2015;451(Pt A):46-64.

24. Tasci Y, Dilbaz B, Uzmez Onal B, et al. The value of cord blood interleukin-6 levels for predicting chorioamnionitis, funisitis and neonatal infection in term premature rupture of membranes. Eur J Obstet Gynecol Reprod Biol 2006;128:34-9.

25. Buck C, Bundschu J, Gallati H, Bartmann P, Pohlandt F. Interleukin-6: a sensitive parameter for the early diagnosis of neonatal bacterial infection. Pediatrics 1994;93:54-8.

26. Shannon E, Noveck R, Sandoval F, Kamath B, Kearney M. Thalidomide suppressed interleukin- 6 but not tumor necrosis factor-alpha in volunteers with experimental endotoxemia. Transl Res 2007;150:275-80.

27. Waage A, Brandtzaeg P, Halstensen A, Kierulf P, Espevik T. The complex pattern of cytokines in serum from patients with meningococcal septic shock. Association between interleukin 6, interleukin 1, and fatal outcome. J Exp Med 1989;169:333-8.

28. Ng PC, Lam HS. Diagnostic markers for neonatal sepsis. Curr Opin Pediatr 2006;18:125-31. 